\title{
Therapeutic targeting of BAG3: considering its complexity in cancer and heart disease
}

\author{
Jonathan A. Kirk, ${ }^{1}$ Joseph Y. Cheung, ${ }^{2}$ and Arthur M. Feldman ${ }^{3}$ \\ 'Department of Cell and Molecular Physiology, Loyola University Chicago, Chicago, Illinois, USA. Division of Renal Medicine, Brigham and Women's Hospital, Boston, Massachusetts, USA. ${ }^{3}$ Department of \\ Medicine, Lewis Katz School of Medicine at Temple University, Philadelphia, Pennsylvania, USA.
}

\begin{abstract}
Bcl2-associated athanogene-3 (BAC3) is expressed ubiquitously in humans, but its levels are highest in the heart, the skeletal muscle, and the central nervous system; it is also elevated in many cancers. BAC3's diverse functions are supported by its multiple protein-protein binding domains, which couple with small and large heat shock proteins, members of the Bcl2 family, other antiapoptotic proteins, and various sarcomere proteins. In the heart, BAC3 inhibits apoptosis, promotes autophagy, couples the $\boldsymbol{\beta}$-adrenergic receptor with the L-type $\mathrm{Ca}^{2+}$ channel, and maintains the structure of the sarcomere. In cancer cells, BAC3 binds to and supports an identical array of prosurvival proteins, and it may represent a therapeutic target. However, the development of strategies to block BAC3 function in cancer cells may be challenging, as they are likely to interfere with the essential roles of BAG3 in the heart. In this Review, we present the current knowledge regarding the biology of this complex protein in the heart and in cancer and suggest several therapeutic options.
\end{abstract}

\section{Introduction}

An important challenge in modern medicine has been that new drugs, biologics, and even vaccines may be precise in modifying the function of a particular target yet still have off-target effects that can hinder their therapeutic development. When the conflict involves proteins that play critical roles in the physiology of the heart but at the same time facilitate the advance of cancer, both caregivers and patients can be challenged. An excellent example can be found in the ongoing development of Bcl2-associated athanogene-3 (BAG3) agonists for the treatment of heart failure (HF) and the development of BAG3 inhibitors for the treatment of cancer.

Cancer cells are highly stressed by metabolic dysfunction, aberrant RNA splicing, accelerated protein synthesis, metabolic reprogramming, and the accumulation of misfolded proteins and other cellular debris. To survive, cancer cells have developed the ability to enhance the activity of the proteasome and the autophagy pathways while simultaneously blocking apoptosis (1). Thus, a logical strategy for ridding the organism of malignancy would be to inhibit protein quality control (PQC) and activate targeted apoptosis (2). Under this strategy, the cellular debris that accumulates in cancer cells would be cleared by BAG3-responsive prosurvival signaling pathways: autophagy, mitophagy, and the ubiquitin-associated proteasomal pathway $(3,4)$. Unfortunately, efforts to develop BAG3-targeted anticancer therapeutics have

Conflict of interest: AMF is the founder of Renovacor Inc. and was a director of the company until it became public in 2021. His conflicts include ownership of equity, income, and research support. He is the inventor on US patent US20170016066A1 and European Union patent W02019237002A1 (Optimization d'une thérapie génique de bag3). All patents have been licensed by Temple University to Renovacor Inc. JYC is an equity holder in Renovacor Inc.

Copyright: (c) 2021, American Society for Clinical Investigation.

Reference information: J Clin Invest. 2021;131(16):e149415

https://doi.org/10.1172/JCI149415. been limited in part by the fact that the heart also requires a robust system of BAG3-dependent PQC. Addressing this type of question served as the foundation for the recent emergence of the field of cardio-oncology and for the recent development of strategies that take advantage of innovations in molecular biochemistry, including gene therapy $(5-7)$.

This therapeutic challenge is best illustrated by the recent development of proteasome inhibitors for the treatment of cancer. One of the first proteasome inhibitors to reach clinical trials was bortezomib, which showed limited ability to modify autophagy flux but blocked the proteasome and upregulated levels of BAG3 $(8,9)$. By contrast, BAG3 knockdown in ex vivo human leukemic cells potentiated bortezomib's induction of apoptosis (9). Carfilzomib, a proteasome inhibitor that does not upregulate BAG3, proved to be significantly better than bortezomib in treating cancer in a large head-to-head comparison $(10,11)$. While the overall incidence of $\mathrm{HF}$ was low in both groups, nearly 3-fold more events were attributed to HF in the carfilzomib group (8.2\%) compared with the bortezomib group (2.8\%).

While BAG3 remains an interesting and important target for new therapies in both cancer and heart disease, it has not been tested in either context. However, recent discoveries regarding both the molecular and the cellular biology of BAG3 raise questions regarding its potential usefulness in cancer because of concerns regarding cardiotoxicity. This discussion will focus attention on two specific diseases that illustrate the therapeutic conundrum in which substantive research relevant to the biology of cancer and heart disease has been carried out: pancreatic ductal adenocarcinoma (PDAC) and hereditary dilated cardiomyopathy with severe heart failure with reduced ejection fraction (HFrEF).

\section{BAG3}

BAG3, its name derived from "athanatos," Greek for "against death," is the third member of a family of six proteins that 


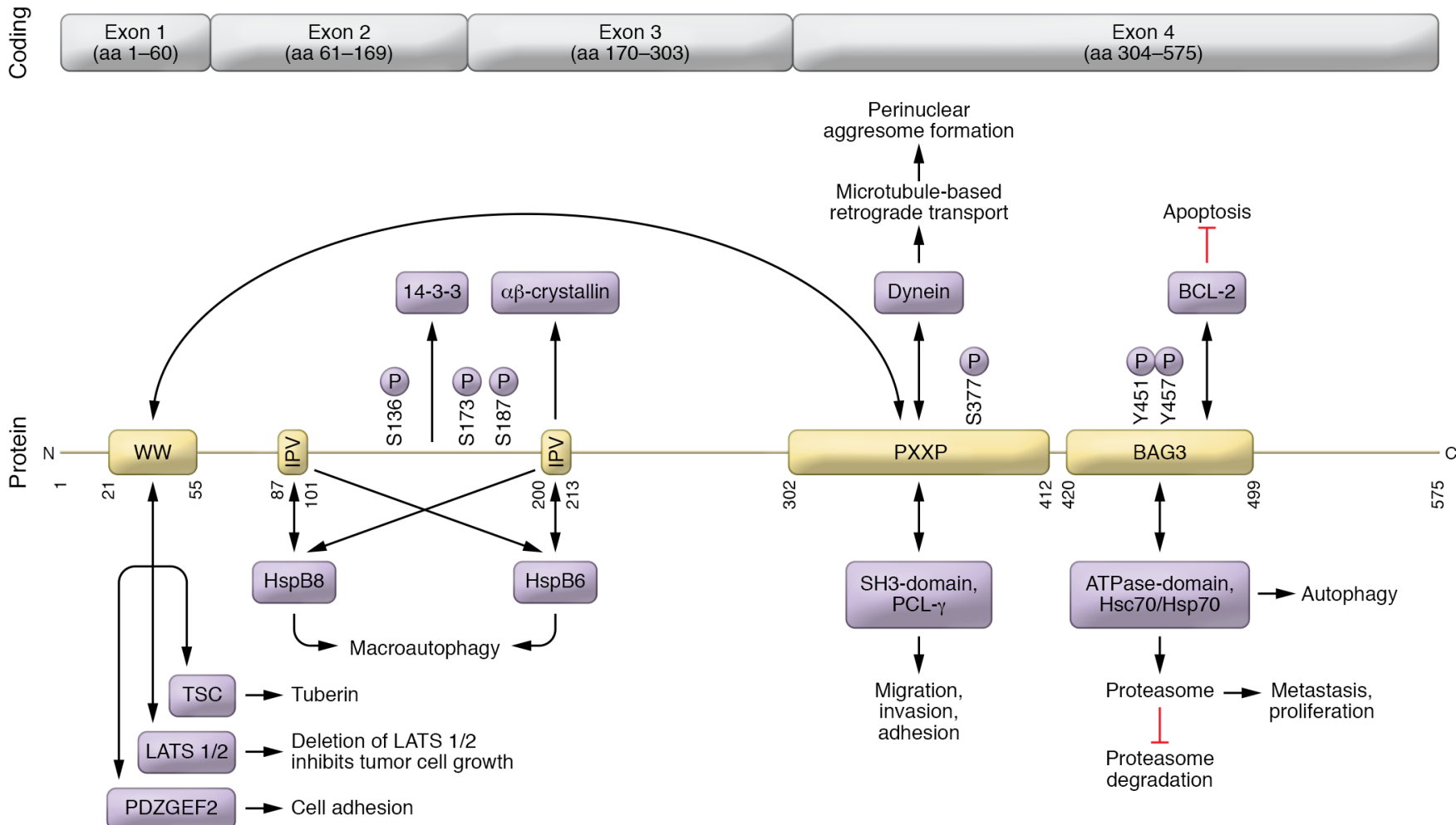

Figure 1. Structure and function of BAG3. Binding sites on BAG3 are shown in yellow, and their binding partners are shown in purple. Posttranslational modifications (specifically phosphorylation) are indicated vertically.

bind to the ATPase motif of heat shock cognate 70 (heat shock protein 70; hsp70) through a sequence in the BAG domain $(12,13)$. That BAG3 plays a critical role in living organisms is supported by the fact that a homolog of BAG3 is expressed in all domains of life, including yeast, invertebrates, amphibians, mammals, and even plants (14). BAG3 is a constitutively expressed, multifunctional, and ubiquitous protein that is most abundant in the heart, skeletal muscle, and central nervous system and in many cancers $(3,15)$. It limits the intrinsic (mitochondria-dependent) pathway of apoptosis by coupling with $\mathrm{Bcl} 2$ and activates macroautophagy by cochaperoning both large and small heat shock proteins. Its expression is induced by stress, proteasome inhibitors, $50 \mathrm{~Hz}$ electromagnetic fields (14), and aging (12, 16-20).

BAG3 translocates to the nucleus, where it can bind to its own promoter and autoregulate its transcription (21). Heat shock transcription factor 1 (HSF1) interacts with two elements in BAG3's promoter to increase its expression in an NF- $\kappa \mathrm{B}-$ dependent manner (22). It also appears to be sensitive to less wellknown transcription factors including the androgen-regulated protein androgen-induced bZIP (AIbZIP) (23), fibroblast growth factor 2 (FGF-2) (24), and early growth response proteins. BAG3 is also expressed in endothelial cells, where its absence leads to decreased angiogenesis (25).

\section{Mechanism of action of BAG3}

BAG3 interacts with a plethora of signaling pathways that would seem unmanageable were it not for the fact that the protein has numerous protein-protein binding domains that support these dif- ferent activities. Moving from the carboxy terminus to the amino terminus of the peptide (Figure 1), the first binding site is the BAG domain, which includes a motif that binds the ATPase site of the cochaperone hsp70. The BAG domain also includes the binding site for $\mathrm{Bcl} 2$, which initiates the canonical signaling cascade that inhibits the intrinsic limb of apoptosis (26). Next, BAG3's PXXP domain has two important partners. First, it binds with proteins that have Src domains, as found in phospholipase C- $\gamma$ or Src, which enhances its ability to regulate cancer cells' adhesion, migration, and invasion of other cells and tissues (27). Second, it serves as the docking station for the motor protein dynein, a microtubule-based minus-end-directed transport system that carries cargo to perinuclear aggresomes for eventual digestion $(16,28)$. There are then two Ile-Pro-Val (IPV) domains that bind the small heat shock proteins hspB6 and hspB8 and provide cellular protection during hypoxia/reoxygenation by binding to the heat shock cognate hsc70 $(29,30)$. Located between the two IPV domains is a binding site for the 14-3-3 protein that targets misfolded proteins to the dynein motor complex (16). The intermediate domain of BAG3 is also a binding site for $\alpha \beta$-crystallin (31). At the amino terminus, the WW domain may interact with proline-rich repeats such as the guanine nucleotide exchange, the pentose base protein, synaptopodin-2, the YAP/TAZ inhibitors LATS1/2 and AMOTL1/2, or the tuberous sclerosis 1 protein $(32,33)$. The WW domain is required for the induction of autophagy in glioma cells (34).

$B A G 3$ and the proteasome. Eukaryotic cells have two primary means of insuring homeostasis and proteostasis: the ubiquitinproteasome system (UPS) and the autophagy-lysosome system. In the UPS, degradation-prone proteins are first ubiquitinated by the 
A Excitation-contraction coupling and sarcomere integrity

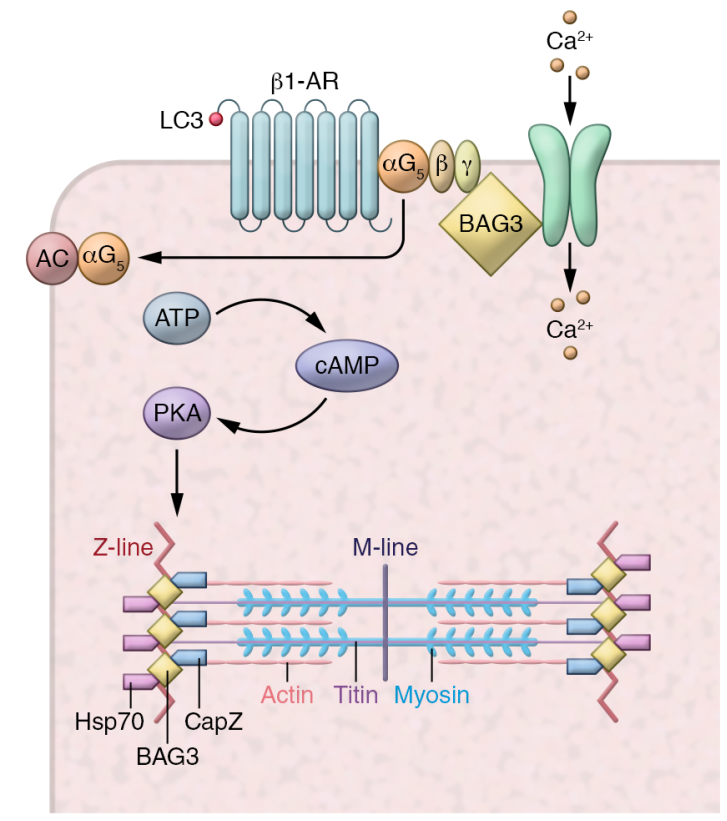

\section{Apoptosis}

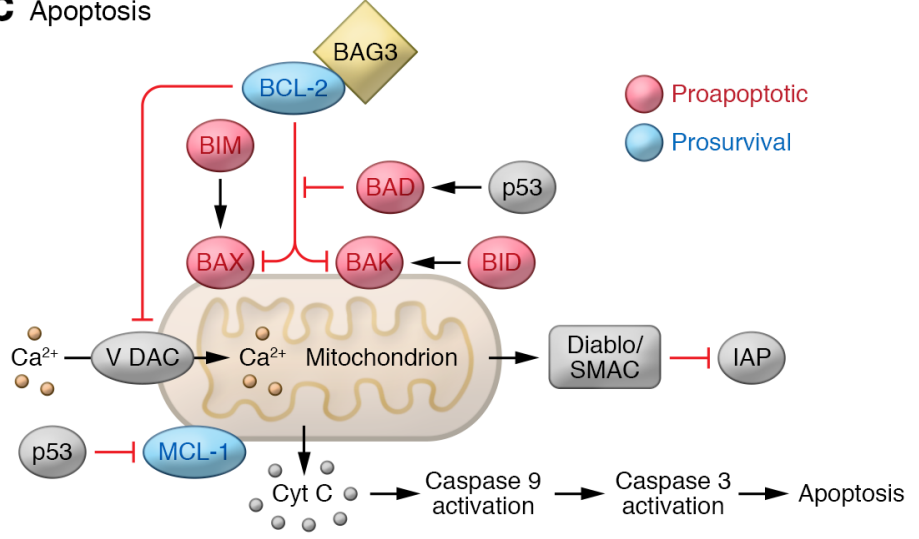

B

Autophagy

Mitophagy
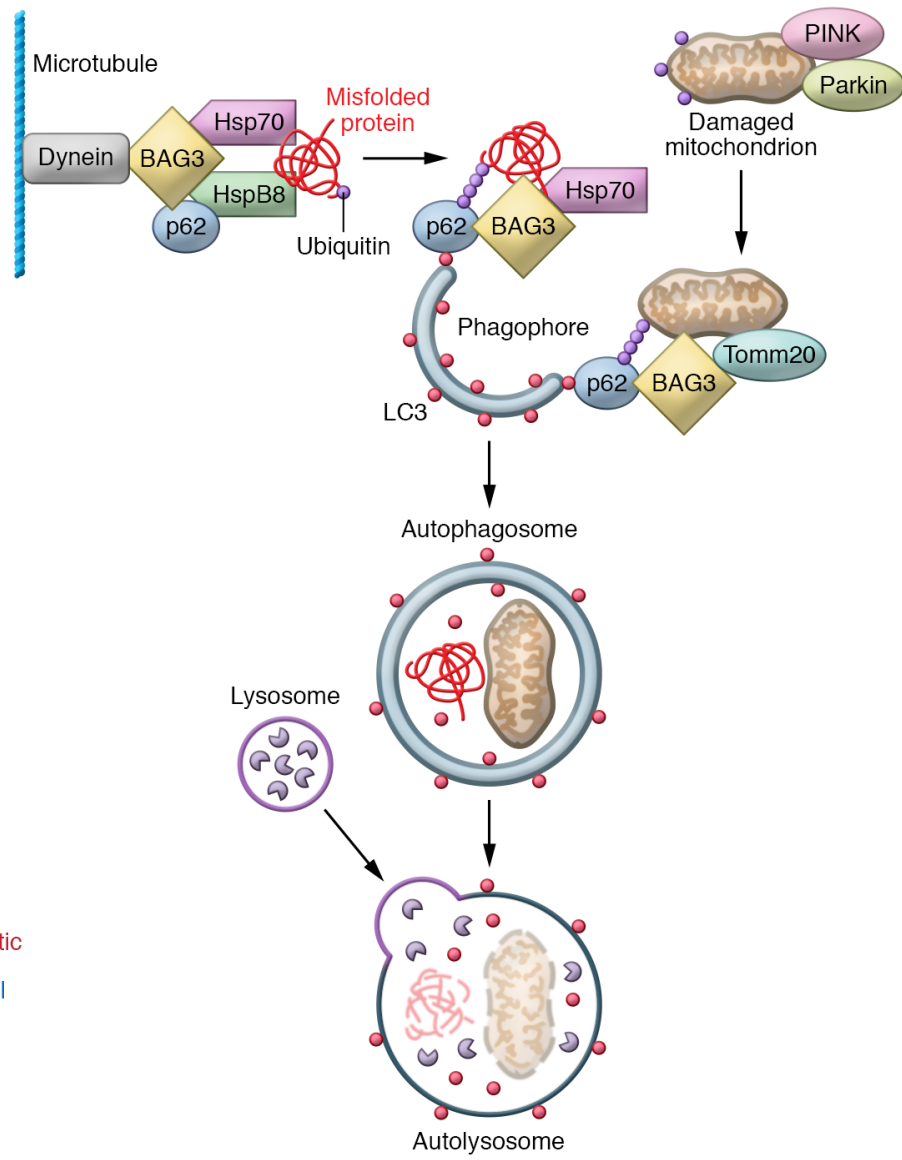

Figure 2. Diverse cellular roles of BAG3. (A) BAG3 regulates contractility in adult ventricular myocytes, colocalizing with $\mathrm{Na}^{+}-\mathrm{K}^{+} / \mathrm{ATPase}$ and the L-type $\mathrm{Ca2}^{+}$channel in the sarcolemma and the transverse tubules (t-tubules). Figure 3 shows BAC3's function in the sarcomere in more detail. (B) BAC3 also plays a central role in autophagy and mitophagy, creating a complex including hsp70 and hspB8 to deliver ubiquitinated, misfolded proteins to the phagophore. This process is both specific and selective. BAG3 is also involved in the maturation of the phagophore into the autophagosome, through its interaction with synaptopodin-2 (SYNPO2) and its associated proteins. BAG3 also serves as an anchoring point for the dynein motor pathway, an intracellular transport system that moves cargo toward the minus ends of microtubules, where they are packaged into perinuclear aggresomes for eventual removal from the cell. (C) Finally, BAC3 couples to BcI2 to limit the mitochondria-dependent apoptosis pathway.

ATP-consuming ubiquitin conjugation machinery. The polyubiquitinated proteins are then degraded by the proteasome, a barrel-shaped and highly conserved multiprotein complex (35). When cells are exposed to stress, they switch from the UPS degradation pathway to the aggresome-lysosome pathways (autophagy) to clear client proteins from the cell. This proteasome-to-autophagy switch is mediated by BAG3 and two proteins that it chaperones: hspB8 and hspA1A.

$B A G 3$ and autophagy. Autophagy is a complex evolutionarily conserved process that clears the cell of debris that arises from cell division, metabolism, and other cell processes (36). A full description of autophagy is outside the scope of this Review; however, in view of the important role that BAG3 plays in autophagy, a brief introduction is provided. The reader is referred to several excellent reviews for additional information $(4,15,37,38)$.
Autophagy is generally divided into three mechanistic groupings: microautophagy, chaperone-mediated autophagy (CMA), and macroautophagy (Figure 2 and ref. 39). Microautophagy is the process in which cytoplasmic debris directly enters a lysosome through an outpouching of the lysosomal membrane. CMA specifically targets substrates with a biochemical motif related to the sequence KFERQ $(40,41)$. Misfolded proteins are unfolded through the action of cytosolic chaperones and then translocated across the lysosomal membrane, where they are digested (42). CMA requires lysosomal-associated membrane protein $2 \mathrm{~A}$ (LAMP2A), hspA8, and hsp90. Macroautophagy is a complex and multistep process in which selective substrates are packaged outside of a lysosome and then transported to the lysosome for degradation (Figure 2). In selective macroautophagy or chaper- 
one-assisted selective autophagy (CASA), the membrane appears to wrap around the cargo in order to fit the specific target $(43,44)$. The CASA machinery, which impacts the processes of adhesion, migration, and proliferation $(44,45)$, consists of synaptopodin-2, the WW domain of BAG3, an autophagosome membrane fusion complex, and YAP/TAZ signaling (16, 46-48). Macroautophagy PQC can be impaired by sustained inflammation (49).

BAG3 and apoptosis. BAG3 inhibits apoptosis in the heart and in some cancers through binding to $\mathrm{Bcl} 2$ with subsequent inhibition of the canonical intrinsic pathway of apoptosis (50). However, $\mathrm{Bcl} 2$ is but one member of the 25 -member Bcl 2 family. Therefore, it is not surprising that noncanonical BAG3-mediated pathways might also play a role. This is supported by studies of $\mathrm{Bcl} 2$ family members in the hematopoietic system, including $\mathrm{Bcl}-\mathrm{x}_{\mathrm{L}}$ and Bax, which have very similar structures and form ion channels in synthetic membranes (51-53) but, as far as we know, are not strongly regulated by $\mathrm{Bcl} 2(54-57)$. The reader is referred to the references for additional details.

\section{BAC3 in cancer}

A decade ago, heat shock proteins were thought to be ideal targets for cancer drug therapy, because their expression was increased in cancer, (58); overexpression could induce tumor-specific apoptosis in vitro (59); and a small-molecule inhibitor of hsp70 potentiated hsp90 inhibitor-induced apoptosis in colon carcinoma cells (60). However, the overall effect of early pharmaceuticals on the treatment of cancer was modest, causing investigators to pivot to the cochaperone of hsp70, BAG3 (61). BAG3 is an ideal target: (a) it can promote survival through multiple cell pathways; (b) its expression is induced by stress and growth factors found in cancer cells; (c) high levels of expression correlate directly with chemoresistance; and (d) high levels of BAG3 predict a poor outcome in a variety of cancers, including thyroid, breast, liver, leukemias, and metastatic melanoma (62-65). BAG3 protects estrogen receptor- $\alpha$-positive neuroblastoma and breast cancer cells through an estrogen response element-independent noncanonical autophagy pathway (66), and also protects non-small cell lung cancer cells from apoptosis (67). However, cancer cells do not follow a script, and in epithelial thyroid cancer cells, knockdown of BAG3 induces epithelial-mesenchymal transition and increased migration and invasion $(62,68)$.

Oncologists, seeing great potential in BAG3 as a therapeutic target, began studies of its mechanism of action in both animal models of cancer and patients with disease. Colvin et al. showed that BAG3 interacts with the SH3 domain of Src, a non-protein tyrosine kinase $(27,69)$. This interaction resulted in enhanced mediation of Src signaling with hsp70 by modulating the activity of transcription factors including NF- $\mathrm{B}$, FoxM1, hypoxia-inducible factor- $1 \alpha$, the translation regulator $\mathrm{HuR}$, and the cell cycle regulators p21 and survivin (27). These studies clearly demonstrated that enhanced coupling of BAG3 and hsp70 supported tumor growth whereas an absence of BAG3-hsp70 had the opposite effect (27). These investigations also identified a small-molecule inhibitor of autophagy, YM-1, which disrupted the hsp70-BAG3 interaction, leading to a loss of Src signaling and to a failure of autophagy to regulate the amount of cellular debris and misfolded proteins (27). These results were confirmed by Li and colleagues, who demon- strated that an allosteric inhibitor of the hsp70-BAG3 proteinprotein interaction had antiproliferative activity across all cancer cell lines tested $(27,61)$.

Another BAG3-related signaling mechanism that has been shown to promote survival of cancer cells is the antiapoptotic $\mathrm{Bcl} 2$ family member Mcl-1, which prevents programmed cell death by binding to proapoptotic members of the family, including Bax and Bak (70). By contrast, BAG3 knockdown reduced the level of the antiapoptotic $\mathrm{Bcl} 2$ protein $\mathrm{Bcl}-\mathrm{x}_{\mathrm{L}}(71-76)$.

Pancreatic cancer. Studies of BAG3 in pancreatic ductal adenocarcinoma (PDAC) provide the best examples of the complexity of using BAG3 as a target for anticancer therapy. Two decades ago, cancer biologists reported for the first time that BAG3 was overexpressed in human pancreatic cancer and in cell lines derived from these tumors (77). By contrast, BAG3 mRNA levels were not elevated in other gastrointestinal cancers, including hepatocellular carcinoma, esophageal, stomach, and colon cancers. The elevation in BAG3 levels in pancreatic cancer was inversely related to survival (78). Pancreatic cancer was also associated with an increase in anti-BAG3 antibodies, which proved useful in discriminating between neoplastic lesions and normal pancreas and between neoplastic lesions and other causes of pancreatic disease such as pancreatitis $(77,78)$. The finding that BAG3 was also detectable in the sera of PDAC patients suggested a role for the secreted form of BAG3 in tumor development (79).

In an important study, Rosati et al. found that not only was BAG3 released from human PDAC cells in culture, but BAG3 could be detected in both the exosome and soluble fractions of PDAC cell lines and colocalized with a cytosolic marker for endosomes (80). These results suggested that BAG3 might be secreted through exosomal pathways. The investigators also found that BAG3 attached to receptors on the surface of macrophages, resulting in their activation and the secretion of PDAC supporting factors. The receptor, identified as interferon-induced transmembrane protein 2 (IFITM-2), signals through the PI3K and the p38 MAP kinase pathways. Notably, BAG3/IFITM-2 binding and the subsequent activation of macrophages were independent of hsp70. The extracellular BAG3 activation of the IFITM-2 receptor on macrophages caused the macrophages to secrete factors that stimulate PDAC cell proliferation. Interruption of macrophage activation leads to decreased tumor growth and metastasis. Importantly, IFITM-2 was required for BAG3 binding and signaling, as its deletion mitigated the untoward effects of BAG3 on macrophage activation. The BAG3 antibody did, however, inhibit tumor growth and metastasis. In aggregate, these studies suggested that the BAG3/ IFITM-2 pathway plays an important role in cellular homeostasis and could be another novel therapeutic target.

Although BAG3 fulfills its canonical role of enhancing autophagy and inhibiting apoptosis in the pancreas, it also has a noncanonical effect in that it supports the reprograming of the malignant cells in the pancreas to aerobic glycolysis so that energy levels can be achieved that will support the rapid growth and reproduction that characterize pancreatic cancer (81). Investigators have also identified another noncanonical pathway in which BAG3 promotes cell invasion by stabilizing IL-8 transcription via HuR recruitment and subsequently suppressing the loading of microRNA-4312 containing the miRNA-induced silencing complex in 
PDAC. This novel pathway links BAG3 expression to enhanced PDAC metastasis (82). In aggregate, these studies support the use of BAG3 as a target for the treatment of patients with PDAC; however, that enthusiasm must be tempered by the recognition that the regulation and function of autophagy in pancreatic cancer are complex and likely require precise targeting (83). Finally, it should be noted that BAG3 controls angiogenesis through regulation of ERK phosphorylation, providing another reason to consider BAG3 as a therapeutic target in cancer (25).

\section{BAC3 and the heart}

BAG3 genetics and genomics. The first evidence that BAG3 plays an important part in cardiac homeostasis and proteostasis came, in retrospect, from a genome-wide association study (GWAS) in 2006 by Patrick Ellinor et al. that identified a novel locus for the phenotype of dilated cardiomyopathy, diffuse myocardial fibrosis, and sudden death on chromosome 10q25-26 (84). In 2011, Norton et al. identified a deletion mutation in BAG3 that segregated with family members with a heritable form of dilated cardiomyopathy (DCM) (85). Subsequent studies of probands from multiple countries with hereditary DCM identified disease-causing coding region mutations, the majority of which were either truncations or deletions (86-88). Interestingly, BAG3 levels were also reduced in $\mathrm{HF}$ patients with nonheritable forms of $\operatorname{DCM}(88,89)$.

Further confirmation that BAG3 deficiency plays an important role in the pathobiology of HFrEF has come from a group of large GWAS and large proband studies that have identified BAG3 variants as one of 2 to 14 genes that can cause heritable DCM BAG3 being the second most common after titin in the majority of studies (90-97).

Important information regarding the clinical presentation of BAG3-related disease came from the European Genetic Cardiomyopathies Initiative, describing the clinical epidemiology in over 100 subjects with defined BAG3 genotypes. Presumptive BAG3 mutations were associated with an $80 \%$ penetrance and an earlier onset of DCM and a more severe disease in men. The authors also noted a prevalence of truncations or deletions that encompassed the BAG domain and a course of the disease that was more aggressive than seen with other heritable forms of DCM (98).

In 2009, Selcen et al. described the cases of three unrelated children with myofibrillar myopathy who harbored a heterozygous single-nucleotide polymorphism in exon 3 of $B A G 3$ that resulted in a substitution of a leucine for a proline at amino acid position 209 (Pro209Leu) (99). Although this disease does not have a name, it is distinguished from other forms of BAG3-related DCM by its unique phenotype: severe and progressive muscle weakness ultimately resulting in respiratory failure, a restrictive/hypertrophic cardiomyopathy, elevated levels of serum creatine kinase, and neurologic abnormalities including giant axon disease. Recently, investigators reported three missense mutations targeting the same proline 209 codon - each causing a unique phenotype: (a) early cardiomyopathy with distal myopathy (Pro209Leu); (b) late Charcot-Marie-Tooth type 2 neuropathy (Pro209Ser); and (c) late distal myopathy (Pro209Gln) (100). All three Pro209 mutants were found to have a toxic gain-of-function mutation that caused the mutant to aggregate hsp70, and interfere with autophagy (100). However, there is ongoing controversy because a mouse model of the variant did not result in a cardiomyopathy phenotype (101). The reader is therefore referred to several excellent reviews of this disease that is seen predominantly in children $(102,103)$.

In the more common BAG3 mutations, coding region insertions, or deletions, patients present with a DCM and a positive family history. We now know that BAG3 variants do not necessarily have to cause DCM but can instead influence the phenotype if disease occurs (104). For example, the presence of any one of four variants in patients of African ancestry was associated with a nearly 2 -fold increase in either death or worsening HF if the individual developed the disease (104).

BAG3 mechanism of action in the heart. The first evidence of BAG3's role in cardiac pathobiology came in 2006 when Homma et al. reported that homozygous deletion of BAG3 in mice led to profound myofibrillar disorganization and death by 4 weeks of age (105). Homma et al. also reported that heterozygotes were normal, but that determination was made at just 4 weeks of age. In 2010, Hishiya et al., using neonatal myocytes in which BAG3 had been knocked down and small cardiac muscle strips from $\mathrm{Bag}^{+/-}$and WT mice, showed that BAG3 and its cochaperone hsp70 stabilized myofibril structure by forming a complex with the F-actin capping protein CAPZ $\beta 1$ (106). In the absence of BAG3, even modest stress led to disruption of the myofibril structures (107). Later studies using mice in which one allele of Bag3 was ablated showed that haploinsufficiency was associated with diminished autophagy and increased apoptosis as well as modest left ventricle (LV) dysfunction (108). By contrast with neonatal cardiac myocytes, in adult myocytes BAG3 was predominantly expressed in the sarcolemma and the t-tubules $(102,108)$. Genetic variants in BAG3 have a similar role in modulating the susceptibility to ischemia in the skeletal musculature, an effect that is strain specific (109).

BAG3 is also cardioprotective during the stress of hypoxia followed by reperfusion (110). When hearts infected with an AAV2/9 vector and the CMV promoter driving the expression of BAG3 underwent exposure to ischemia/reperfusion injury, AAV2/9-BAG3 pretreatment significantly protected the heart. These salutary effects of BAG3 were attributable in part to decreased apoptosis, increased autophagy, and increased autophagic flux (110). BAG3 gene therapy also ameliorated the decrease in LV function seen in hearts after left anterior descending coronary artery occlusion when AAV9-BAG3 was administered two months after the infarction (111). These beneficial effects of BAG3 were accompanied by improved cell shortening, enhanced systolic $\left[\mathrm{Ca}^{2+}\right]_{\mathrm{i}}$, increased transients in $\left[\mathrm{Ca}^{2+}\right]_{\mathrm{i}}$ amplitudes, and increased maximal L-type $\mathrm{Ca}^{2+}$ current amplitude (112).

To model BAG3 cardiomyopathy in a human system, Judge et al. generated an isogenic series of human induced pluripotent stem cells (iPSCs) with loss-of-function mutations in BAG3 (113). These heterozygous mutations demonstrated reduced protein expression, disrupted myofibril structure, and compromised contractile function. The studies also found that hspB8 is a particularly important partner for BAG3 in cardiomyocytes. Mass spectroscopy suggested that the primary interactions occurred with proteins associated with PQC, ribosomal/RNA binding, nuclear proteins, and secreted proteins (113). However, Judge et al. also reported that the accumulation of ubiquitinated protein after bortezomib treatment was the same in WT and BAG3-deficient cardiomyo- 


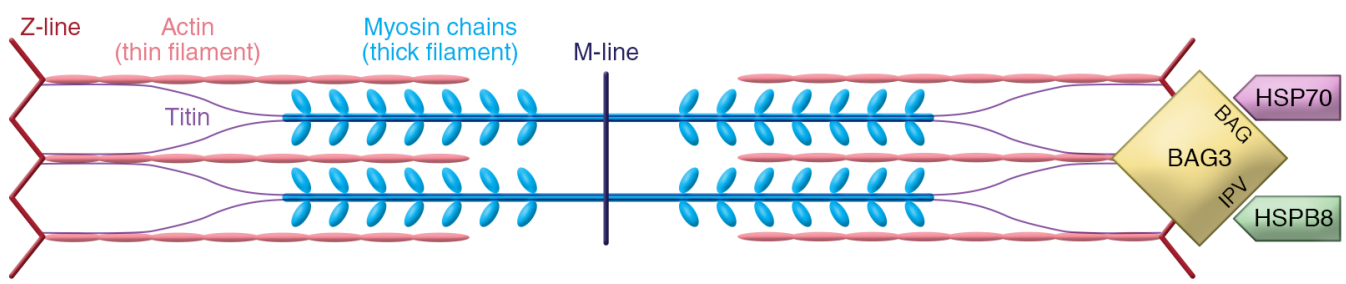

Healthy heart

Normal sarcomere BAG3 levels Removal of mechanical stressinduced misfolded proteins Normal function

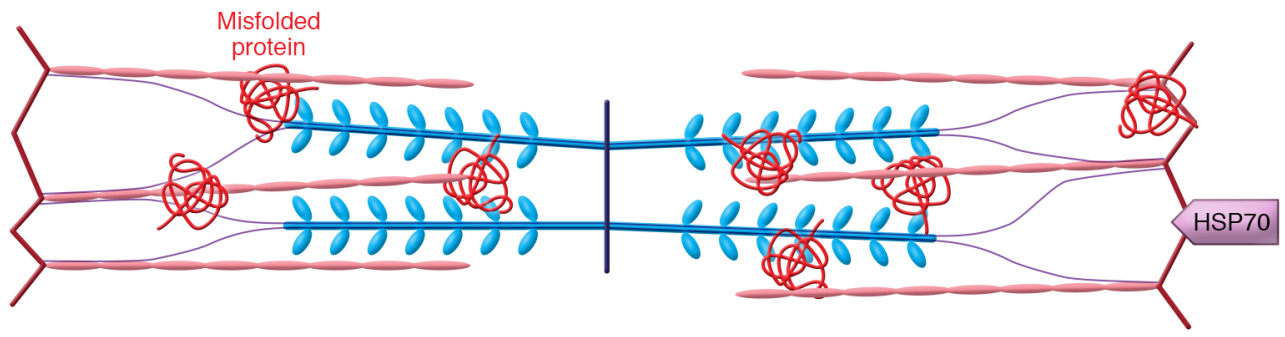

Heart failure Low sarcomere BAG3 levels Accumulation of ubiquitinated proteins in sarcomere lattice Reduced function

Figure 3. Sarcomere protein quality control requires BAG3. In the normal heart, the BAG3-hsp70-hspB8 complex localizes to the Z-disc, where it provides chaperone-assisted selective autophagy for sarcomere proteins, which helps to maintain normal contractile function. However, in heart failure (specifically heart failure with reduced ejection fraction, HFrEF), sarcomere-localized BAC3 levels drop, and thus ubiquitinated proteins are not removed and instead stay imbedded in the sarcomere lattice structure. These nuclear, stress-misfolded proteins contribute to the cellular contractile dysfunction observed in heart failure.

cytes, suggesting that BAG3 is not required for the regulation of bulk autophagy flux.

Investigators have taken advantage of transgenic technology to evaluate the mechanism by which a point mutation or the loss of a single allele of BAG3 results in DCM (114). Knockin of the human mutation $\mathrm{BAG}^{\mathrm{E} 455 \mathrm{~K}}$ disrupted the interaction between BAG3 and hsp70 and resulted in a DCM phenotype (114). In both the BAG3-knockout and E455K-knockin mouse, the levels of small heat shock proteins were diminished whereas a subset of proteins that maintain cardiac homeostasis were elevated $(114,115)$. Similarly, cardiac-specific knockouts of one allele of BAG3 also led to a substantial decrease in LV function and cardiac dilatation that were associated with a decrease in small heat shock proteins (114). Other investigators using a similar cardiac-restricted knockout model reported that the decrease in LV function was associated with a decrease in autophagy and autophagy flux as well as a significant increase in myocardial apoptosis (112). Mice in which the ascending aorta had been banded with eventual development of cardiac dilatation and LV dysfunction and mice harboring muscle LIM protein (MLP) knockout showed a 50\% reduction in BAG3 levels, consistent with studies in the human heart (88). Thus, diminished levels of BAG3 appear to be a ubiquitous response to stress in the heart.

Another interesting response to stress was reported by De Marco and colleagues. Using mass spectroscopy to measure BAG3 levels in sera, they found for the first time that BAG3 was released from the heart in patients with DCM and stress (116). BAG3 release was associated with an increase in immune response, as anti-BAG3 antibodies were also measurable in the serum of these patients. However, the release of BAG3 was seen only in patients with class IV symptoms, indicative of severe disease.

$B A G 3$ regulates contractility in the adult heart. In adult ventricular myocytes, BAG3 is colocalized with $\mathrm{Na}^{+} / \mathrm{K}^{+}$-ATPase and the L-type $\mathrm{Ca}^{2+}$ channel in the sarcolemma and the t-tubules (108). In fact, BAG3 coimmunoprecipitated with the $\beta_{1}$-adren- ergic receptor, the L-type $\mathrm{Ca}^{2+}$ channels, and phospholemman (108). A reduction in maximal calcium-activated force $\left(\mathrm{F}_{\max }\right)$ generated by the myofilament is a hallmark of HFrEF in both humans and animal models $(117,118)$. While the cause of this decline was not known, it was suspected that it arose from structural disorganization and reduced sarcomeric PQC, which is a poorly understood process in the adult heart (119). However, recent work has shown that the BAG3-hsp70-hspB8 complex localizes to the sarcomere to mediate the turnover of a number of clients, such as tropomyosin, myosin light chain, and filamin C (120). In human $\mathrm{HF}$, the decrease in $\mathrm{F}_{\text {max }}$ directly correlated with reduced myofilament and localized BAG3 levels. Heterozygous deletion of Bag3 in mice resulted in a similar phenotype, and AAV-driven restoration of BAG3 in a mouse HF model rescued myofilament function and PQC (Figure 3).

The levels of BAG3 in the human heart during $\mathrm{HF}$ are not influenced by either age or sex. However, myofilament localization of BAG3 was significantly decreased in cardiac tissue from males with DCM but not in heart tissue from females (121). This difference was independent of estrogen but was closely correlated with changes in LV heat shock transcription factor 1 (HSF1). Specifically, the myofilament-associated HSF1 protein decreases in HF.

BAG3 also plays an important role in modulating the homeostasis of the mitochondria (122). Mitochondrial disruption can result in failure to generate enough ATP and/or the unfettered release of reactive oxygen species, leading to increased apoptosis (123). BAG3 and parkin are recruited to depolarized mitochondria, where they promote mitophagy, whereas suppression of BAG3 significantly reduced autophagy flux and eliminates clearance of TOM20, an essential import receptor for maintaining appropriate levels of mitochondrial proteins (124).

To investigate how a DCM-causing mutation $(\mathrm{R} 477 \mathrm{H})$ could impact myofibrillar organization and the chaperone network in cardiomyocytes, McDermott-Roe et al. generated isogenic genome-edited human iPSC-derived cardiomyocytes (iPSC- 
CMs) (125). Although normal at baseline, fiber length and alignment declined markedly in $\mathrm{R} 477 \mathrm{H}$ and knockout iPSC-CMs following proteasome inhibition with MG132. Proteasome inhibition increased BAG3 and P62 expression in healthy cells, but in mutant cells it caused fiber redistribution, fiber disarray, accumulation of ubiquitinated proteins, and enhanced expression of the autophagy pathway proteins BAG3, P62, and LC3II.

Connexin $43(\mathrm{Cx} 43)$ is a critical protein in the heart that can rapidly change its function by altering the gap junction and slowing cell-cell communication. Early studies suggested that impairment of the autophagy-lysosome pathway degraded $\mathrm{Cx} 43$ by either inhibiting lysosomal activity or diminishing the level of BAG3 (126). Inhibition of lysosomal activity enhanced accumulation of Cx43 aggregates and suppressed BAG3 turnover. Knockdown of BAG3 dysregulated $\mathrm{Cx} 43$ protein degradation and decreased the levels of $\mathrm{Cx} 43$ by changing the stability of the protein. BAG3 can also signal the accumulation of $\mathrm{Cx} 43$, suggesting that BAG3 plays a role in $\mathrm{Cx} 43$ turnover under normal circumstances and during stress. In fact, when cells were starved, there was a reduction in levels of Cx43-P1 and Cx43-P2, forms of Cx43 that may reflect posttranslational (phosphorylation) modifications. Knockdown of BAG3 reduced levels of $\mathrm{Cx} 43$ by dysregulating $\mathrm{Cx} 43$ protein stability, and it impaired lysosomal turnover of $\mathrm{Cx} 43$.

Based on recent work by Xi Fang, we also know that small heat shock proteins closely monitor protein folding for a variety of tissues, including the heart (127). Specifically, they mediate chaperone functions by interacting with BAG3 at the two IPV motifs independent of the actions of the BAG, WW, and PXXP domains. However, the presence of hsp70 may be necessary for these interactions to occur (114). Elevated levels of hspB8 have been found in both animal models and patients with both acute and chronic cardiovascular disease, including acute and chronic myocardial infarction, chronic myocardial ischemia, and pressure overloadinduced cardiac hypertrophy.

Targeting BAG3: protecting the heart while treating cancer. To illustrate the complexity that may arise in treating life-threatening illnesses with agents that lack tissue specificity, we sought examples of a pair of diseases where the therapeutic goal was to inhibit a specific protein to treat one of the diseases but the therapeutic goal for the second disease was to stimulate that same pathway. We also looked for diseases that affected large numbers of patients, that had substantial morbidity and/or mortality, and for which the pharmacologic effects of the therapy were not organ specific. HF and lung cancer were ideal for illustrating this challenge. Both affect large numbers of patients: HF is the most common diagnosis at the time of discharge in individuals over the age of 65 who are hospitalized in the United States, and its incidence is increasing. Lung cancer is the second most common form of cancer, following prostate cancer in men and breast cancer in women. Although the rate of lung cancer has decreased and survival improved for men, the opposite is true for women (128).

From a teleological perspective, BAG3 is unique in that it serves multiple purposes that vary depending on cell type, as opposed to other proteins that do the same thing in multiple cells. In fact, BAG3's multiple roles in cell survival appear to make its therapeutic reduction more complicated. In fact, in the heart, BAG3 maintains tissue homeostasis and proteo- stasis through the very same pathways by which it supports the growth and survival of hematopoietic cancers and solid tumors - pro-autophagy and antiapoptosis. However, the studies with bortezomib and carfilzomib provide an important clue: cardiac toxicity was seen in only a very small percentage of subjects receiving either of these two agents.

The results of the bortezomib-carfilzomib studies described above led us to hypothesize that under normal situations, systemic pharmacologic inhibition of BAG3 is not robust enough to alter cardiac function because of inherent redundancy in the autophagy and apoptosis signaling pathways. This hypothesis is supported by the finding that when the proteasome is inhibited, BAG3 and the heat shock proteins can pivot and transport cargo to aggresomes and lysosomal autophagic pathways. Similarly, if the endogenous pathway of apoptosis is inhibited, the cell can pivot and utilize the exogenous (mitochondria-independent) pathway or ship misfolded proteins as appropriate to the proteasome. The theory that loss-of-function genetic mutations in cardioprotective pathways can be associated with a higher incidence of side effects of targeted anticancer therapy is supported by the demonstration of a carfilzomib-sensitive PDAC subgroup with a specific transcriptomic phenotype that could explain the group's increased sensitivity to the agent (129). This means of interpreting the effectiveness of anticancer therapies and their risk to noncancerous cells and tissues based on genotype is a novel concept, but one that that has gained recent attention (130).

Significant advances in gene therapy and innovations in delivery systems including viral vectors with enhanced tropism, vectors with diminished immunogenicity, and gene therapy that can be delivered on a chip or by other novel delivery systems may also be helpful in preventing new chemotherapeutic agents from causing cardiotoxicity in patients who are at increased risk. We believe that we could learn a great deal more about cardiotoxicity in anticancer therapy and whether there are potential opportunities to mitigate off-target effects if oncologists begin to genotype both the tumor and the person who harbors the tumor (130). Similarly, cardiologists need to begin to genotype patients with new-onset DCM as we continue to recognize new genetic causes of this common disease. A recent report suggests that important information regarding target identification may also be gleaned from new and novel serum biomarkers (131). One potential biomarker is TNF- $\alpha$, which activates the death receptor pathway, which in turn may lead to cell survival, apoptosis, or a regulated form of necrosis (108). The signaling pathway can then travel alternative paths, activating cell survival, apoptosis, or necrosis. A major decision point is the choice between apoptosis or necrosis - a choice predicated on the activity of caspases (132).

Several clinical points regarding the potential of gene therapy also merit mention. First, because the expression of the transfected gene is often driven by a viral promoter, there is a theoretical concern that "overexpression" could have undue consequences. However, in the case of BAG3, the gene self-regulates, and therefore, endogenous expression should turn off if levels get too high (21). Second, BAG3, when driven by an AAV9 vector, does not integrate into the genome and thus is not passed on to daughter cells during cell division. The corollary of this observation is that therapeutic BAG3 does not serve as an oncogene. 
Third, in patients with single-nucleotide polymorphisms that are not amenable to traditional gene replacement therapy because of potential dominant-negative effects, CRISPR/Cas9 may soon be used to correct functional single-nucleotide variants. And finally, while this Review has focused on the heart and cancer, we would be disingenuous if we did not report that an increasing number of studies suggest that targeting BAG3 may also be effective in the treatment of diseases attributable to abnormalities in PQC in the central nervous system, including Huntington's disease, amyotrophic lateral sclerosis, Parkinson's disease, and Alzheimer's disease (133-135). The development of a BAG3-targeted gene therapy for neurodegenerative diseases is an exciting new area in the field of PQC and regulation of apoptosis. By contrast with cancer, neurodegenerative disease requires adequate levels of
BAG3 consistent with the myocardium. Research is also under way in a hunt for small molecules that might target BAG3 and increase its expression. Regardless of approach, BAG3 remains an enticing target for therapeutic gene and drug discovery.

\section{Acknowledgments}

This work was supported by NIH grants HL136737 to JAK and HL91799 and HL12309 to AMF and a grant from Renovacor Inc. to AMF.

Address correspondence to: Arthur M. Feldman, Lewis Katz School of Medicine at Temple University, 3440 North Broad Street, Suite 200, Philadelphia, Pennsylvania 19140, USA. Phone: 215.718.7231; Email: arthur.feldman@tuhs.temple.edu.
1. Nandi $D$, et al. The ubiquitin-proteasome system.

JBiosci. 2006;31(1):137-155.

2. Chen Y, et al. Proteasome dysregulation in human cancer: implications for clinical therapies. Cancer Metastasis Rev. 2017;36(4):703-716.

3. Behl C. Breaking BAG: the co-chaperone BAG3 in health and disease. Trends Pharmacol Sci. 2016;37(8):672-688.

4. Kogel D, et al. At the crossroads of apoptosis and autophagy: multiple roles of the co-chaperone BAG3 in stress and therapy resistance of cancer. Cells. 2020;9(3):574.

5. Narayan V, Ky B. Common cardiovascular complications of cancer therapy: epidemiology, risk prediction, and prevention. Anпи Rev Med. 2018;69:97-111.

6. Hamo CE, et al. Cancer therapy-related cardiac dysfunction and heart failure. Part 2: Prevention, treatment, guidelines, and future directions. Circ Heart Fail. 2016;9(2):e002843.

7. Ganatra S, et al. Management of cardiovascular disease during coronavirus disease (COVID-19) pandemic. Trends Cardiovasc Med. 2020;30(6):315-325.

8. Du ZX, et al. Caspase-dependent cleavage of BAG3 in proteasome inhibitors-induced apoptosis in thyroid cancer cells. Biochem Biophys Res Commun. 2008;369(3):894-898.

9. Liu P, et al. BAG3 gene silencing sensitizes leukemic cells to Bortezomib-induced apoptosis. FEBS Lett. 2009;583(2):401-406.

10. Dimopoulos MA, et al. Carfilzomib and dexamethasone versus bortezomib and dexamethasone for patients with relapsed or refractory multiple myeloma (ENDEAVOR): a randomised, phase 3, open-label, multicentre study. Lancet Oncol. 2016;17:27-38.

11. Dimopoulos MA, et al. Carfilozomib versus bortezomib for relapsed or refractory myeloma authors' reply. Lancet Oncol. 2016;17:e126.

12. Takayama S, et al. An evolutionarily conserved family of Hsp70/Hsc70 molecular chaperone regulators. J Biol Chem. 1999;274(2):781-786.

13. Lee JH, et al. Bis, a Bcl-2-binding protein that synergizes with Bcl-2 in preventing cell death. Oncogene. 1999;18(46):6183-6190.

14. Rosati A, et al. BAG3: a multifaceted protein that regulates major cell pathways. Cell Death Dis. 2011;2:e141.
15. Marzullo L, et al. The multiple activities of BAG3 protein: mechanisms. Biochim Biophys Acta Gen Subj. 2020;1864(8):129628.

16. Gamerdinger M, et al. BAG3 mediates chaperone-based aggresome-targeting and selective autophagy of misfolded proteins. EMBO Rep. 2011;12(2):149-156.

17. Thress $\mathrm{K}$, et al. Scythe: a novel reaper-binding apoptotic regulator. EMBO J.1998;17(21):6135-6143.

18. Moribe Y, et al. Samui, a novel cold-inducible gene, encoding a protein with a BAG domain similar to silencer of death domains (SODD/ BAG-4), isolated from Bombyx diapause eggs. Eur JBiochem. 2001;268(12):3432-3442.

19. Briknarova K, et al. Structural analysis of BAG1 cochaperone and its interactions with $\mathrm{Hsc70}$ heat shock protein. Nat Struct Biol. 2001;8(4):349-352.

20. Takayama S, Reed JC. Molecular chaperone targeting and regulation by BAG family proteins. Nat Cell Biol. 2001;3(10):E237-E241.

21. Gentilella A, Khalili K. Autoregulation of co-chaperone BAG3 gene transcription. JCell Biochem. 2009;108(5):1117-1124.

22. Franceschelli S, et al. Bag3 gene expression is regulated by heat shock factor 1. J Cell Physiol. 2008;215(3):575-577.

23. Qi H, et al. AIbZIP, a novel bZIP gene located on chromosome 1q21.3 that is highly expressed in prostate tumors and of which the expression is up-regulated by androgens in LNCaP human prostate cancer cells. Cancer Res. 2002;62(3):721-733.

24. Gentilella A, Khalili K. BAG3 expression is sustained by FGF 2 in neural progenitor cells and impacts cell proliferation. Cell Cycle. 2010;9(20):4245-4247.

25. Falco A, et al. BAG3 controls angiogenesis through regulation of ERK phosphorylation. Oncogene. 2012;31(50):5153-5161.

26. Chao DT, Korsmeyer SJ. BCL-2 family: regulators of cell death. Annu Rev Immunol. 1998;16:395-419.

27. Colvin TA, et al. Hsp70-Bag3 interactions regulate cancer-related signaling networks. Cancer Res. 2014;74(17):4731-4740.

28. Kassis JN, et al. CAIR-1/BAG-3 modulates cell adhesion and migration by downregulating activity of focal adhesion proteins. Exp Cell Res. 2006;312(15):2962-2971.

29. Ni E, et al. The PXXP domain is critical for the protective effect of BAG3 in cardiomyocytes. Clin Exp Pharmacol Physiol. 2019;46(5):435-443.

30. Carra S. The stress-inducible HspB8-Bag3 complex induces the eIF2alpha kinase pathway: implications for protein quality control and viral factory degradation? Autophagy. 2009;5(3):428-429.

31. Hishiya A, et al. BAG3 directly interacts with mutated alphaB-crystallin to suppress its aggregation and toxicity. PLoS One. 2011;6(3):e16828.

32. Gout E, et al. Co-chaperone BAG3 and adenovirus penton base protein partnership. J Cell Biochem. 2010;111(3):699-708.

33. Ulbricht A, et al. Cellular mechanotransduction relies on tension-induced and chaperone-assisted autophagy. Curr Biol. 2013;23(5):430-435.

34. Merabova N, et al. WW domain of BAG3 is required for the induction of autophagy in glioma cells. J Cell Physiol. 2015;230(4):831-841.

35. Saeki Y, Tanaka K. Assembly and function of the proteasome. Methods Mol Biol. 2012;832:315-337.

36. Parzych KR, Klionsky DJ. An overview of autophagy: morphology, mechanism, and regulation. Antioxid Redox Signal. 2014;20(3):460-473.

37. Hiebel C, et al. BAG3 proteomic signature under proteostasis stress. Cells. 2020;9(11):E2416.

38. Giudice A, et al. Review of molecular mechanisms involved in the activation of the Nrf2-ARE signaling pathway by chemopreventive agents. Methods Mol Biol. 2010;647:37-74.

39. Sturner E, Behl C. The role of the multifunctional BAG3 protein in cellular protein quality control and in disease. Front Mol Neurosci. 2017;10:177.

40. Park JS, et al. Regulation of amyloid precursor protein processing by its KFERQ motif. BMB Rep. 2016;49(6):337-342.

41. Dice JF. Peptide sequences that target cytosolic proteins for lysosomal proteolysis. Trends Biochem Sci. 1990;15(8):305-309.

42. Orenstein SJ, Cuervo AM. Chaperone-mediated autophagy: molecular mechanisms and physiological relevance. Semin Cell Dev Biol. 2010;21(7):719-726.

43. Mijaljica D, et al. The intriguing life of autophagosomes. Int J Mol Sci. 2012;13(3):3618-3635.

44. Yorimitsu T, Klionsky DJ. Autophagy: molecular machinery for self-eating. Cell Death Differ. 2005;12(suppl 2):1542-1552.

45. Hosokawa N, et al. Atg101, a novel mammalian autophagy protein interacting with Atg13. Auto- 
phagy. 2009;5(7):973-979.

46. Gamerdinger M, et al. Protein quality control during aging involves recruitment of the macroautophagy pathway by BAG3. EMBO J. 2009;28(7):889-901.

47. Carra S, et al. HspB8 participates in protein quality control by a non-chaperone-like mechanism that requires eIF2\{alpha\} phosphorylation. J Biol Chem. 2009;284(9):5523-5532.

48. Gamerdinger M, et al. Emerging roles of molecular chaperones and co-chaperones in selective autophagy: focus on BAG proteins. J Mol Med (Berl). 2011;89(12):1175-1182.

49. Hartupee J, et al. Impaired protein quality control during left ventricular remodeling in mice with cardiac restricted overexpression of tumor necrosis factor. Circ Heart Fail. 2017;10(12):e004252.

50. Haudek SB, et al. TNF provokes cardiomyocyte apoptosis and cardiac remodeling through activation of multiple cell death pathways. JClin Invest. 2007;117(9):2692-2701.

51. Minn AJ, et al. Bcl-x(L) forms an ion channel in synthetic lipid membranes. Nature. 1997;385(6614):353-357.

52. Antonsson B, et al. Inhibition of Bax channelforming activity by Bcl-2. Science. 1997;277(5324):370-372.

53. Suzuki M, et al. Structure of Bax: coregulation of dimer formation and intracellular localization. Cell. 2000;103(4):645-654.

54. Eskes R, et al. Bid induces the oligomerization and insertion of Bax into the outer mitochondrial membrane. Mol Cell Biol. 2000;20(3):929-935.

55. Wei MC, et al. tBID, a membrane-targeted death ligand, oligomerizes BAK to release cytochrome c. Genes Dev. 2000;14(16):2060-2071.

56. Sionov RV, et al. Regulation of bim in health and disease. Oncotarget. 2015;6(27):23058-23134.

57. Schenk RL, et al. Characterisation of mice lacking all functional isoforms of the pro-survival BCL-2 family member A1 reveals minor defects in the haematopoietic compartment. Cell Death Differ. 2017;24(3):534-545.

58. Wisen S, et al. Binding of a small molecule at a protein-protein interface regulates the chaperone activity of hsp70-hsp40. ACS Chem Biol. 2010;5(6):611-622.

59. Powers MV, et al. Dual targeting of HSC7O and HSP72 inhibits HSP9O function and induces tumor-specific apoptosis. Cancer Cell. 2008;14(3):250-262.

60. Massey AJ, et al. A novel, small molecule inhibitor of Hsc70/Hsp70 potentiates Hsp90 inhibitor induced apoptosis in HCT116 colon carcinoma cells. Cancer Chemother Pharmacol. 2010;66(3):535-545.

61. Li X, et al. Validation of the Hsp70-Bag3 protein-protein interaction as a potential therapeutic target in cancer. Mol Cancer Ther. 2015;14(3):642-648.

62. Meng X, et al. Knockdown of BAG3 induces epithelial-mesenchymal transition in thyroid cancer cells through ZEB1 activation. Cell Death Dis. 2014;5:e1092.

63. Shields S, et al. BAG3 promotes tumour cell proliferation by regulating EGFR signal transduction pathways in triple negative breast cancer. Oncotarget. 2018;9(21):15673-15690.
64. Xiao H, et al. BAG3 and HIF- $1 \alpha$ coexpression detected by immunohistochemistry correlated with prognosis in hepatocellular carcinoma after liver transplantation. Biomed Res Int. 2014;2014:516518.

65. Guerriero L, et al. BAG3 protein expression in melanoma metastatic lymph nodes correlates with patients' survival. Cell Death Dis. 2014;5:e1173.

66. Felzen V, et al. Estrogen receptor $\alpha$ regulates non-canonical autophagy that provides stress resistance to neuroblastoma and breast cancer cells and involves BAG3 function. Cell Death Dis. 2015;6:e1812.

67. Zhang Y, et al. Bag3 promotes resistance to apoptosis through Bcl-2 family members in non-small cell lung cancer. Oncol Rep. 2012;27(1):109-113.

68. Li N, et al. PKC $\delta$-mediated phosphorylation of BAG3 at Ser187 site induces epithelialmesenchymal transition and enhances invasiveness in thyroid cancer FRO cells. Oncogene. 2013;32(38):4539-4548.

69. Colvin TA, et al. Proteotoxicity is not the reason for the dependence of cancer cells on the major chaperone Hsp70. Cell Cycle. 2014;13(14):2306-2310.

70. Boiani $\mathrm{M}$, et al. The stress protein BAG3 stabilizes Mcl-1 protein and promotes survival of cancer cells and resistance to antagonist ABT-737. J Biol Chem. 2013;288(10):6980-6990.

71. Jacobs AT, Marnett LJ. HSF1-mediated BAG3 expression attenuates apoptosis in 4-hydroxynonenal-treated colon cancer cells via stabilization of anti-apoptotic $\mathrm{Bcl}-2$ proteins. J Biol Chem. 2009;284(14):9176-9183.

72. Sugio A, et al. BAG3 upregulates Mcl-1 through downregulation of miR-29b to induce anticancer drug resistance in ovarian cancer. Gynecol Oncol. 2014;134(3):615-623.

73. Verdecchia A, et al. Recent cancer survival in Europe: a 2000-02 period analysis of EUROCARE-4 data. Lancet Oncol. 2007;8(9):784-796.

74. Pacini F, et al. European consensus for the management of patients with differentiated thyroid carcinoma of the follicular epithelium. Eur JEndocrinol. 2006;154(6):787-803.

75. Nucera C, Pontecorvi A. Clinical outcome, role of $\operatorname{BRAF}(\mathrm{V} 600 \mathrm{E})$, and molecular pathways in papillary thyroid microcarcinoma: is it an indolent cancer or an early stage of papillary thyroid cancer? Front Endocrinol (Lausanne). 2012;3:33.

76. Chiappetta G, et al. BAG3 down-modulation reduces anaplastic thyroid tumor growth by enhancing proteasome-mediated degradation of BRAF protein. JClin Endocrinol Metab. 2012;97(1):E115-E120.

77. Liao $\mathrm{Q}$, et al. The anti-apoptotic protein BAG-3 is overexpressed in pancreatic cancer and induced by heat stress in pancreatic cancer cell lines. FEBS Lett. 2001;503(2-3):151-157.

78. Rosati A, et al. Expression of the antiapoptotic protein BAG3 is a feature of pancreatic adenocarcino$\mathrm{ma}$ and its overexpression is associated with poorer survival. Am J Pathol. 2012;181(5):1524-1529.

79. Falco A, et al. BAG3 is a novel serum biomarker for pancreatic adenocarcinomas. Am J Gastroenterol. 2013;108(7):1178-1180.
80. Rosati A, et al. BAG3 promotes pancreatic ductal adenocarcinoma growth by activating stromal macrophages. Nat Commun. 2015;6:8695.

81. Cairns RA, et al. Regulation of cancer cell metabolism. Nat Rev Cancer. 2011;11(2):85-95.

82. Li C, et al. BAG3 regulates stability of IL-8 mRNA via interplay between HuR and miR-4312 in PDACs. Cell Death Dis. 2018;9(9):863.

83. Li J, et al. Regulation and function of autophagy in pancreatic cancer [published online November 20, 2020]. Autophagy. https://doi.org/10.1080/1 5548627.2020.1847462.

84. Ellinor PT, et al. A novel locus for dilated cardiomyopathy, diffuse myocardial fibrosis, and sudden death on chromosome 10q25-26. J Am Coll Cardiol. 2006;48(1):106-111.

85. Norton N, et al. Genome-wide studies of copy number variation and exome sequencing identify rare variants in BAG3 as a cause of dilated cardiomyopathy. Am J Hum Genet. 2011;88(3):273-282.

86. Chami N, et al. Nonsense mutations in BAG3 are associated with early-onset dilated cardiomyopathy in French Canadians. Can J Cardiol. 2014;30(12):1655-1661.

87. Franaszczyk M, et al. The BAG3 gene variants in Polish patients with dilated cardiomyopathy: four novel mutations and a genotype-phenotype correlation.J Transl Med. 2014;12:192.

88. Feldman AM, et al. Decreased levels of BAG3 in a family with a rare variant and in idiopathic dilated cardiomyopathy. J Cell Physiol. 2014;229(11):1697-1702.

89. Toro R, et al. Familial dilated cardiomyopathy caused by a novel frameshift in the BAG3 gene. PLoS One. 2016;11(7):e0158730.

90. Shah S, et al. Genome-wide association and Mendelian randomisation analysis provide insights into the pathogenesis of heart failure. Nat Commun. 2020;11(1):163.

91. Esslinger U, et al. Exome-wide association study reveals novel susceptibility genes to sporadic dilated cardiomyopathy. PLoS One. 2017;12(3):e0172995.

92. Mazzarotto F, et al. Reevaluating the genetic contribution of monogenic dilated cardiomyopathy. Circulation. 2020;141(5):387-398.

93. Choquet $\mathrm{H}$, et al. Meta-analysis of 26638 individuals identifies two genetic loci associated with left ventricular ejection fraction. Circ Genom Precis Med. 2020;13(4):e002804.

94. Aung N, et al. Genome-wide analysis of left ventricular image-derived phenotypes identifies fourteen loci associated with cardiac morphogenesis and heart failure development. Circulation. 2019;140(16):1318-1330.

95. Jordan E, et al. An evidence-based assessment of genes in dilated cardiomyopathy [published online May 5, 2021]. Circulation. https://doi. org/10.1161/circulationaha.120.053033.

96. Morales A, et al. Variant interpretation for dilated cardiomyopathy: refinement of the American College of Medical Genetics and Genomics/ClinGen guidelines for the DCM Precision Medicine Study. Circ Genom Precis Med. 2020;13(2):e002480.

97. de Denus S, et al. A genetic association study of heart failure: more evidence for the role of BAG3 
in idiopathic dilated cardiomyopathy. ESC Heart Fail. 2020;7(6):4384-4389.

98. Dominguez F, et al. Dilated cardiomyopathy due to BLC2-associated athanogene 3 (BAG3) mutations. J Am Coll Cardiol. 2018;72(20):2471-2481.

99. Selcen D, et al. Mutation in BAG3 causes severe dominant childhood muscular dystrophy. Ann Neurol. 2009;65(1):83-89.

100.Adriaenssens E, et al. BAG3 Pro209 mutants associated with myopathy and neuropathy relocate chaperones of the CASA-complex to aggresomes. Sci Rep. 2020;10(1):8755.

101.Fang X, et al. P209L mutation in Bag3 does not cause cardiomyopathy in mice. Am J Physiol Heart Circ Physiol. 2019;316(2):H392-H399.

102. Meister-Broekema M, et al. Myopathy associated BAG3 mutations lead to protein aggregation by stalling Hsp70 networks. Nat Commun. 2018;9(1):5342.

103. Quintana MT, et al. Cardiomyocyte-specific human Bcl2-associated anthanogene 3 P209L expression induces mitochondrial fragmentation, Bcl2-associated anthanogene 3 haploinsufficiency, and activates p38 signaling. Am J Pathol. 2016;186(8):1989-2007.

104.Myers VD, et al. Association of variants in BAG3 with cardiomyopathy outcomes in African American individuals. JAMA Cardiol. 2018;3(10):929-938.

105. Homma S, et al. BAG3 deficiency results in fulminant myopathy and early lethality. $\mathrm{Am} \mathrm{J}$ Pathol. 2006;169(3):761-773.

106. Hishiya A, et al. BAG3 and Hsc70 interact with actin capping protein CapZ to maintain myofibrillar integrity under mechanical stress. Circ Res. 2010;107(10):1220-1231.

107. Iwasaki M, et al. BAG3 directly associates with guanine nucleotide exchange factor of Rap1, PDZGEF2, and regulates cell adhesion. Biochem Biophys Res Commun. 2010;400(3):413-418.

108. Feldman AM, et al. BAG3 regulates contractility and $\mathrm{Ca}(2+)$ homeostasis in adult mouse ventricular myocytes. J Mol Cell Cardiol. 2016;92:10-20.

109. McClung JM, et al. BAG3 (Bcl-2-associated athanogene-3) coding variant in mice determines susceptibility to ischemic limb muscle myopathy by directing autophagy. Circulation . 2017;136(3):281-296.
110.Su F, et al. Bcl-2-associated athanogene 3 protects the heart from ischemia/reperfusion injury. JCI Insight. 2016;1(19):e90931.

111. Knezevic T, et al. Adeno-associated virus serotype 9-driven expression of BAG3 improves left ventricular function in murine hearts with left ventricular dysfunction secondary to a myocardial infarction. JACC Basic Transl Sci. 2016;1(7):647-656.

112. Myers VD, et al. Haplo-insufficiency of Bcl2associated athanogene 3 in mice results in progressive left ventricular dysfunction, $\beta$-adrenergic insensitivity, and increased apoptosis. J Cell Physiol. 2018;233(9):6319-6326.

113. Judge LM, et al. A BAG3 chaperone complex maintains cardiomyocyte function during proteotoxic stress. JCI Insight. 2017;2(14):e94623.

114. Fang X, et al. Loss-of-function mutations in co-chaperone BAG3 destabilize small HSPs and cause cardiomyopathy. JClin Invest. 2017;127(8):3189-3200.

115. Villard E, et al. A genome-wide association study identifies two loci associated with heart failure due to dilated cardiomyopathy. Eur Heart J 2011;32(9):1065-1076.

116. De Marco M, et al. Detection of soluble BAG3 and anti-BAG3 antibodies in patients with chronic heart failure. Cell Death Dis. 2013;4:e495.

117. Kirk JA, et al. Pacemaker-induced transient asynchrony suppresses heart failure progression. Sci Transl Med. 2015;7(319):319ra207.

118. Blair CA, et al. Heart failure in humans reduces contractile force in myocardium from both ventricles. JACC Basic Transl Sci. 2020;5(8):786-798.

119. Martin TG, Kirk JA. Under construction: the dynamic assembly, maintenance, and degradation of the cardiac sarcomere. J Mol Cell Cardiol. 2020;148:89-102.

120. Martin TG, et al. Cardiomyocyte contractile impairment in heart failure results from reduced BAG3-mediated sarcomeric protein turnover. Nat Commun. 2021;12(1):2942.

121. Martin TG, et al. BAG3 expression and sarcomere localization in the human heart are linked to HSF-1 and are differentially affected by sex and disease. Am J Physiol Heart Circ Physiol. 2021;320(6):H2339-H2350.

122.Palikaras K, Tavernarakis N. Mitochondrial homeostasis: the interplay between mitophagy and mitochondrial biogenesis. Exp Gerontol. 2014;56:182-188.

123. Selivanov VA, et al. Reactive oxygen species production by forward and reverse electron fluxes in the mitochondrial respiratory chain. PLoS Comput Biol. 2011;7(3):e1001115.

124. Tahrir FG, et al. Evidence for the role of BAG3 in mitochondrial quality control in cardiomyocytes. J Cell Physiol. 2017;232(4):797-805.

125. McDermott-Roe C, et al. Investigation of a dilated cardiomyopathy-associated variant in BAG3 using genome-edited iPSC-derived cardiomyocytes. JCI Insight. 2019;4(22):e128799.

126.Ghasemi Tahrir F, et al. Role of Bcl2-associated athanogene 3 in turnover of gap junction protein, connexin 43, in neonatal cardiomyocytes. Sci Rep. 2019;9(1):7658.

127. Fang X, et al. The BAG3-dependent and -independent roles of cardiac small heat shock proteins. JCI Insight. 2019;4(4):e126464.

128. Siegel RL, et al. Cancer statistics, 2021. CA Cancer J Clin. 2021;71(1):7-33.

129. Fraunhoffer NA, et al. Evidencing a pancreatic ductal adenocarcinoma subpopulation sensitive to the proteasome inhibitor carfilzomib. Clin Cancer Res. 2020;26(20):5506-5519.

130. Garcia-Pavia P, et al. Genetic variants associated with cancer therapy-induced cardiomyopathy. Circulation. 2019;140(1):31-41.

131. Narayan V, et al. Mechanistic biomarkers informative of both cancer and cardiovascular disease: JACC state-of-the-art review. J Am Coll Cardiol. 2020;75(21):2726-2737.

132. Guo X, et al. Cardioprotective role of tumor necrosis factor receptor-associated factor 2 by suppressing apoptosis and necroptosis. Circulation. 2017;136(8):729-742.

133. Cristofani R, et al. A crucial role for the protein quality control system in motor neuron diseases. Front Aging Neurosci. 2020;12:191.

134. Ravikumar B, et al. Inhibition of mTOR induces autophagy and reduces toxicity of polyglutamine expansions in fly and mouse models of Huntington disease. Nat Genet. 2004;36(6):585-595.

135. Lei Z, et al. BAG3 facilitates the clearance of endogenous tau in primary neurons. Neurobiol Aging. 2015;36(1):241-248. 